\title{
Contexto psicossocial de mulheres mastectomizadas
}

\author{
Psychosocial context of mastectomized women
}

\section{Ruth Silva Lima da Costa ${ }^{1}$, Elizângela Pessoa Pereira², Emily Assis Tavares³, Gleicy Janáira da Costa Queiroz ${ }^{4}$, Juliana Quinteiro5, Mirtes da Silva Andrade Ribeiro ${ }^{6}$}

\begin{abstract}
'Autora para correspondência. Centro Universitário Uninorte. Rio Branco, Acre, Brasil. ORCID: 0000-0003-1890-086X. ruttylyma@gmail.com ${ }^{2}$ Centro Universitário Uninorte. Rio Branco, Acre, Brasil. ORCID: 0000-0001-6019-8862. elly.pessoa@gmail.com ${ }^{3}$ Centro Universitário Uninorte. Rio Branco, Acre, Brasil. ORCID: 0000-0002-8511-4060. emily.assistavares@hotmail.com ${ }^{4}$ Centro Universitário Uninorte. Rio Branco, Acre, Brasil. ORCID: 0000-0002-9202-7205. gleicynaira123@gmail.com ${ }^{5}$ Centro Universitário Uninorte. Rio Branco, Acre, Brasil. ORCID: 0000-0001-9901-1604. julianaquinteiro46@gmail.com ${ }^{6}$ Centro Universitário Uninorte. Rio Branco, Acre, Brasil. ORCID: 0000-0003-4806-9261. ribeiromirtes@hotmail.com
\end{abstract}

RESUMO I OBJETIVO: Conhecer os aspectos psicossociais de mulheres mastectomizadas. MÉTODO: Trata-se de estudo de abordagem qualitativa, realizado com 12 mulheres mastectomizadas e acompanhadas em uma unidade de assistência de alta complexidade de tratamento de câncer do Acre. RESULTADOS: Os resultados apontam que a maioria das mulheres eram casadas $5(42 \%)$, com idade entre 50 a 55 anos 7 (58\%), com nível médio completo 4 (33\%) e renda familiar entre 1 a 2 salários mínimos 8 (67\%). Os achados foram agrupados em quatro categorias: 0 enfrentamento da mastectomia, as dificuldades enfrentadas pela mulher após a cirurgia, a interferência da mastectomia nos seus relacionamentos e a relação da mesma com a sua autoimagem e autoestima. Conforme os relatos, para algumas mulheres a vivência frente a mastectomia foi tranquila, no entanto para outras foi um processo que gerou sentimentos de tristeza e medo do novo. Houve repercussões negativas, relacionamentos familiares afetados, incapacidade no funcionamento social e alteração na imagem corporal. Para elas, o apoio familiar foi fundamental para o enfrentamento da nova realidade. CONCLUSÃo: Realizar o procedimento afeta a vida emocional das mulheres, sendo assim, tornase indispensável que elas recebam um adequado suporte psicológico principalmente por parte da família e dos profissionais de saúde.
ABSTRACT | OBJECTIVES: To know the psychosocial aspects of mastectomized women. METHODS: This is a qualitative study, conducted with 12 mastectomized women and followed up in a high complexity care unit for the treatment of cancer in Acre. RESULTS: The results indicate that most women were married $5(42 \%)$, aged 50 to 55 years 7 (58\%), with complete average level 4 (33\%) and family income between 1 and 2 minimum wages 8 (67\%). The findings were grouped into four categories: Facing mastectomy, difficulties faced by women after surgery, interference mastectomy in their relationships and its relationship with the your self-image and self-esteem. According to reports, for some women the experience of mastectomy was quiet, however for others it was a process that generated feelings of sadness and fear of the new. There were negative repercussions, affected family relationships, disability in social functioning and altered body image. For them, family support was fundamental for facing the new reality. CONCLUSION: Performing the procedure affects the emotional life of women, so it is essential that they receive adequate psychological support especially from family and health professionals.

KEYWORDS: Breast Cancer. Mastectomy. Psychosocial.

PALAVRAS-CHAVE: Câncer de mama. Mastectomia. Psicossocial. 


\section{Introdução}

O câncer de mama é a segunda neoplasia mais comum entre as mulheres no Brasil e no mundo. Estima-se que em 2018 houve no Brasil em torno de 59.700 casos novos representando uma taxa de incidência de 51,29 casos a cada 100.000 mulheres ${ }^{1}$.

Culturalmente os seios são fontes de diversas simbologias: desde a antiguidade pinturas e esculturas os destacavam como uma expressão de maternidade, sinônimo de feminilidade, representação sensual e do prazer no íntimo e até como símbolo de empoderamento feminino em protestos históricos, no entanto, eles estão suscetíveis a uma das patologias que mais matam mulheres por neoplasias no Brasil: O câncer de mama².

Nesse contexto, o câncer de mama entra como um grande desafio a ser superado pela mulher após seu diagnóstico, pois a neoplasia vai além da sua dimensão física, uma vez que a mama representa um símbolo de feminilidade, ele irá também afetá-la em seus aspectos emocionais e sociais ${ }^{3}$.

A mastectomia, excisão ou remoção parcial ou total da mama, insere-se neste contexto como um dos principais e mais utilizados meios de tratamento e busca da cura do câncer de mama, porém ao mesmo tempo em que se trata de uma potencial solução, é tida como um grande prejuízo na vida da mulher, havendo grandes repercussões físicas e psicológicas, necessitando de uma base familiar estruturada com amparo e apoio mútuo ${ }^{4}$.

Frente a diagnóstico de câncer e a necessidade da realização da mastectomia, a mulher pode vivenciar um caminho desconhecido com grandes dificuldades, que podem vir acompanhadas por sentimentos, como angústia, tristeza, o medo da morte e da mutilação ${ }^{5}$.

O enfrentamento da doença é complexo, pois há uma mudança drástica na vida da mulher, já ela terá que enfrentar diversos obstáculos, desafios e dificuldades que exigirão dela adaptações em todo seu cotidiano ${ }^{6}$.

Há um grande impacto que costuma afetar as muIheres que passam pela mastectomia, principalmente no que se refere aos aspectos emocionais.
São sentimentos divergentes no tocante ao processo cirúrgico e prognóstico da doença. No entanto, a família, os amigos, os parceiros, a fé e crenças religiosas são elementos cruciais para que elas se sintam seguras e fortalecidas frente ao restabelecimento da sua autoestima7.

A assistência a essas mulheres é de suma importância nesse processo, onde a escuta qualificada de suas queixas e o apoio emocional com abordagem individualizada, em uma ação conjunta de toda a equipe multidisciplinar, terá importante papel durante toda a terapêutica ${ }^{8}$.

Nesse sentido, o presente estudo teve como objetivo de conhecer os aspectos psicossociais de mulheres mastectomizadas.

\section{Métodos}

Trata-se de estudo de abordagem qualitativa, com direcionamento exploratório e descritivo, realizado com 12 mulheres mastectomizadas e acompanhadas em unidade de assistência de alta complexidade de tratamento de câncer do Acre.

A amostra se deu a partir do número total de muIheres que realizaram mastectomia no ano de 2017 em Rio Branco - Acre. Do total de 20 mulheres que realizaram a cirurgia de mastectomia no período, foram selecionadas pelo método de seleção aleatória simples, 12 mulheres para participaram do presente estudo.

Foram utilizados os seguintes critérios de inclusão: mulheres mastectomizadas no ano de 2017 em Rio Branco - Acre; aceitarem participar da pesquisa a partir da assinatura do TCLE (Termo de Consentimento Livre e Esclarecido). Foram excluídas as mulheres mastectomizadas no mesmo período, mas que não eram provenientes do município de Rio Branco.

Os dados de identificação das mulheres foram colhidos através das informações inseridas em seus prontuários. Após essa fase, os pesquisadores entraram em contato prévio com as mesmas por via telefônica e marcaram a melhor data e o melhor horário para a realização da coleta de dados. 
As entrevistas ocorreram no domicílio das mulheres, tiveram uma duração em média de 40 minutos e foram realizadas por toda a equipe de pesquisa que foi previamente treinada pela pesquisadora responsável do estudo. O objetivo do treinamento foi garantir a confidencialidade dos dados coletados sem a influência de percepções pessoais e garantir o suporte emocional as mulheres, caso fosse necessário, durante a realização da coleta de dados.

Foi elaborado um roteiro com as perguntas norteadoras da entrevista que teve início apenas após a leitura explicativa e assinatura do TCLE. A conversação foi gravada e, posteriormente, transcrita na íntegra em arquivo eletrônico.

A análise das entrevistas foi realizada pela categorização de dados: Primeiramente foi realizado a ordenação dos dados obtidos na entrevista; seguido da classificação dos mesmos com a leitura exaustiva e repetida dos textos, estabelecendo interrogações para identificar informações relevantes. Esses dados foram então organizados em categorias específicas e feitas uma análise final com a articulação entre os dados encontrados e os referenciais teóricos da pesquisa, respondendo às questões da mesma, com base em seus objetivos.

O tratamento dos dados foi feito através da análise qualitativa de conteúdo, compondo-se de leituras flutuantes, emergindo núcleos de significados no conjunto do material coletado, para organização e análise dos dados. O método de análise de conteúdo, enquanto método analítico trata-se de um "conjunto de instrumentos metodológicos cada vez mais sutis em constante aperfeiçoamento, que se aplicam a 'discursos' (conteúdos e continentes) extremamente diversificados"

Com o intuito de preservar a identidade das participantes, de acordo com a Resolução 466/12 que trata da pesquisa com seres humanos, foi utilizada o nome de flores nas citações das falas mesmas na descrição dos resultados, a saber: Rosa, Margarida, Lírio, Tulipa, Violeta, Jacinto, Jasmim Girassol, Orquídea, Hortência, Íris e Erva-Doce.

A pesquisa foi aprovada pelo Comitê de Ética e pesquisa do Centro Universitário Uninorte sob o parecer de $n^{\circ}$ 3.096.167 como também pelo Comitê de Ética e Pesquisa do Hospital das Clínicas do Acre - HCA/ FUNDHACRE sob parecer de aprovação n ${ }^{\circ} 3.235 .844$ e CAAE: 99389218.4.3001.5009.

\section{Resultados e Discussão}

Participaram do estudo 12 mulheres em sua maioria na faixa etária de 50 a 55 anos 7 (58\%), casadas 5 (42\%), com nível médio completo 4(33\%) e com renda familiar entre um e dois salários mínimos 8 (67\%).

De acordo com dados do Instituto Nacional do Câncer (INCA $)^{10}$ o risco de desenvolver o câncer cresce progressivamente com o passar da idade, em especial a partir dos 50 anos que se configura como um dos mais importantes fatores de risco para o câncer de mama devido a pessoa apresentar-se mais exposta às alterações biológicas do envelhecimento, o que corrobora com os achados do presente estudo. Quanto ao estado civil, o estudo de Frutuoso et al. (2009)11 encontrou resultados semelhantes onde $69 \%$ das pacientes mastectomizadas também eram casadas.

Enfrentar todo o processo de diagnóstico de câncer e retirada da mama pode tornar-se muito difícil para algumas mulheres, nesse caso o fato de a maioria delas serem casadas, configura-se como um fato importante, pois segundo o estudo de Gasparelo et al. $(2010)^{12}$, a presença e o apoio de um companheiro, após esse processo, são essenciais para as mulheres durante todas as etapas do tratamento.

Concernente a questão do grau de escolaridade, o estudo de Ramos et al. (2012) identificou que o baixo nível de escolaridade constitui um fator de risco para o desenvolvimento do câncer de mama, pois influencia no acesso destas mulheres a serviços de saúde, em práticas de autocuidado e na adoção de medidas de detecção precoce para a doença ${ }^{13}$. Estudo com 18 mulheres mastectomizadas no município de Belém do Pará, evidenciou que $94 \%$ delas encontravam-se também com renda familiar baixa ${ }^{14}$, o que corrobora com os nossos achados. O mesmo autor enfatiza ainda que o baixo nível socioeconômico é um fator de risco que torna muitas mulheres suscetíveis ao desenvolvimento de doenças, tendo em vista uma maior dificuldade de acesso à programas de promoção de saúde e prevenção primária.

Mediante a análise dos depoimentos, os achados foram agrupados em quatro categorias: a vivência e o enfrentamento do processo de mastectomia, as dificuldades enfrentadas pela mulher após a cirurgia, a interferência da mastectomia nos seus relacionamentos e a relação da mastectomia com a sua autoimagem e autoestima. 


\section{Categoria 01: A vivência e o enfrentamento do processo de mastectomia}

Para algumas mulheres a vivência frente à mastectomia foi tranquila, no entanto para outras foi um processo que gerou sentimentos negativos de tristeza e medo do enfrentamento do novo. As falas a seguir expõem os sentimentos:

Eu não tive problemas, eu assim, eu tava tranquila em relação a esse tipo de coisa, eu vejo algumas colegas disse que chorava muito, eu não, tranquilo, não tive reação assim de se apavorar não, agora eu acho que

Deus me preparou muito pra isso (Rosa, 42 anos).

Não, eu reagi bem, você acredita que eu não parei pra olha com ficou, encarei com naturalidade mesmo, ser natural pra mim, pra mim o importante era a saúde né

(Margarida, 52 anos).

Senti muita dor, tristeza, fiquei com medo (Lírio, 36 anos).

Teve uma vez que eu caí na real, quando eu cheguei em casa com 2 dias que eu me olhei no espelho ne, ai eu chorei, chorei, mas meu marido e minha família conversou muito comigo e eu acabei aceitando.

(Violeta, 53 anos)

O que se observa em relação às mulheres são sentimentos variados quando houve a necessidade da realização do procedimento. As falas supracitadas ressaltam que algumas mulheres aceitaram facilmente a perda da mama, porém, esta resignação se alia, sobretudo, ao fato de que o procedimento cirúrgico ser algo que não pôde ser evitado, sendo a única possibilidade de cura. No entanto para outras o enfrentamento da realidade pareceu ser muito mais difícil, é o que retrata o estudo de Rocha et al. (2019), ao pesquisar os sentimentos de mulheres submetidas a mastectomia total, onde observou-se que emergiram sentimentos divergentes entre as mulheres no tocante ao processo de mastectomia ${ }^{7}$.

Os achados de Vale, Dias e Miranda (2017), ressaltam que a mulher submetida à mastectomia carece de um suporte familiar e social efetivos para a manutenção da qualidade de vida, com a finalidade de ajudá-la na sua reinserção à vida anterior à doença, além de auxiliar no resgate das atividades cotidianas, ao abranger atividades de lazer, retorno ao mercado de trabalho e autoestima ${ }^{15}$.
Em muitos casos após a realização do procedimento, algumas pacientes referem sentir-se mais tranquilas, o que pode estar ligado ao fato do afastamento do medo da morte ocasionado pelo diagnóstico do câncer de mama, é o que afirma o estudo de Fireman et al. ( 2019), sobre a percepção da funcionalidade e qualidade de vida após a mastectomia, onde a maioria da mulheres relataram que após o procedimento melhoraram sua capacidade funcional, emocional e autoestima, possibilitando sua reinserção social e retorno às atividades de vida diária ${ }^{16}$.

\section{Categoria 02: As dificuldades enfrentadas pela mulher após a cirurgia de mastectomia}

Nesta categoria algumas mulheres relatam que o processo foi doloroso, algumas tiveram algumas perdas e outras se mostraram conformadas com a realidade:

Meu marido eu perdi ne? [...], ele não me valorizou, porque geralmente os homi quer as mulher sadia ne, se saber que tem algum problema de saúde já não... eu sofri muito, assim, devido isso ai ne, mas eu me confiei, me peguei em Deus mermo, não foi fácil, porque minha vida não e fácil, porque nem os filhos que a gente tem eles não tem tempo pra ta ali com a gente não. (Jacinto, 45 anos)

Nenhuma. É gente, é aquela coisa, não adianta a gente reclamar, dizer ah meu Deus porque eu retirei meu peito, eu, meu peito tão assim e eu ter tirado, porque, não, se Deus quis né, foi, então. (Jasmim, 73 anos)

Eu penso como vou sobreviver a esses dez anos de tamoxifeno, porque por eu ser muito nova ele preferiu que eu tomasse todo esse tempo, e eu tinha esperança

de tomar só 5, ter chance de engravidar daqui 5, 6 anos. E quando ele disse que seriam 10 anos, isso pra mim foi um tapa na cara, foi mais difícil ir na consulta

ir ouvir isso que seria 10 anos do que a consulta que ouvir que iria fazer quimioterapia. (Erva Doce, 28 anos)

O que se observa em relação às entrevistadas é que frente a realização do procedimento, algumas enfrentaram dificuldades que dentre elas destacam-se: as relações conjugais, a própria aceitação do corpo sem a mama e as limitações físicas. De acordo com Ferreira e Mamede (2013), umas das primeiras dificuldades a serem enfrentadas pela maioria das muIheres após a mastectomia, é sua própria aceitação, como de olhar no espelho e aceitar que seu corpo está diferente, sem uma parte, que culturalmente representa a sua feminilidade ${ }^{17}$. 
Em alguns casos, a necessidade da realização da mastectomia é uma condição que ocasiona tensão, temor, angústias e muita preocupação, e que pode causar muita dor e sofrimento para a mulher e seus familiares, pois acarreta em uma mutilação que repercute em sua imagem corporal, afeta a sua sexualidade e a autoimagem ${ }^{18}$.

A cirurgia pode levar ainda uma série de consequências de ordem física e emocional, podendo influenciar em seus hábitos de vida, provocando alterações nas suas relações, quase sempre provenientes de sentimentos de impotência e de frustração, sobre algo que foge ao seu controle ${ }^{19}$.

Os achados de Almeida et al. (2015) ao analisar a vivencia da mulher jovem com câncer de mama e mastectomizada, identificou que frente as dificuldades vivenciadas por elas, as mesmas procuram buscar sempre motivos que lhes tragam força e apoio para enfrentar essa fase, principalmente os voltados a espiritualida$\mathrm{de}^{20}$.

\section{Categoria 03: A interferência da mastectomia nos relacionamentos da mulher}

As falas a seguir refletem se o procedimento afetou ou não os relacionamentos da mulher, enfatizando o quanto o apoio familiar é importante para o enfrentamento da nova realidade:

[...] bem, é até engraçado, porque eu sempre usei muito sutiã, e eu acho que mudou no começo, mas quando a mulher tá em tratamento corta toda a vontade de sexo, mas o meu marido é uma pessoa muito compreensiva, sempre me deixou bem à vontade. (Violeta, 53 anos).

Não, não afetou em nada não, nada, nada, nada... É uma coisa, eu acho assim, a pessoa tem que se conscientizar, eu penso assim, [...], se Deus permitiu que tirasse minha mama é porque ele não quer mais a gente com aquilo né, se aquele pedaço de carne tá me fazendo mal porque que eu vou ficar com ele, não. (Girassol, 58 anos)
Quando descobri que ia ficar sem a mama, eu chamei meu esposo pra se separar, porque eu imaginei, não posso ficar casada estando careca e sem a mama, tinha que ficar sozinha mesmo. Foi isso que eu pensei, mas meu esposo falou: não, eu casei com você pra gente é pra vida inteira, eu não casei com você só por causa de seus peitos e seus cabelos, a gente vai continuar junto, e eu sinto o mesmo amor sem diferença. Meu esposo me ama eu não tenho dúvidas, minhas filhas também são muito preocupadas comigo. Eu e meu esposo agente leva uma vida normal. (Iris, 50 anos)

Olha eles tem sido $100 \%$, principalmente meus filhos, eu só tenho um casal de filhos né, eles não me largam, dão muito apoio, me dão força, tudo eles fazem pra mim.

(Orquídea, 56 anos)

O que se observa em relação às entrevistadas, são que frente a realização da mastectomia, elas receberam apoio da família o que é fundamental para o enfrentamento de todo o processo, pois lidar com uma mulher mastectomizada torna-se um grande desafio para a família, uma vez que esse processo envolve um relacionamento interpessoal para compreender o significado da doença e suas influências no âmbito físico e emocional ${ }^{21}$.

É possível denominar esta nova fase de processo de readequação da sexualidade, sendo que é muito importante que à sexualidade da mesma seja o foco de atenção da equipe multiprofissional, pois é fundamental o acompanhamento deste aspecto, pois tende a facilitar a interação e integração da mulher, no sentido de atribuir novos significados, entender o próprio corpo e sua identidade feminina a partir dos procedimentos adotados que lhe conferem a manutenção de suas inter-relações ${ }^{22}$.

No que diz respeito ao suporte familiar e social, a família é de extrema importância em todo o processo de enfrentamento ${ }^{23}$, bem como os amigos e os profissionais que vão atender às novas necessidades que irão surgir no decorrer da situação, como os seus cuidados da saúde e o ambiente social, sendo, portanto, os fatores mais importantes na rede de apoio a paciente ${ }^{24}$. 


\section{Categoria 04: A relação da mastectomia com a autoimagem e autoestima da mulher}

As falas a seguir refletem sobre as possíveis modificações ocorridas na vida dessas mulheres no que se refere às repercussões psicológicas, relacionamento familiar, funcionamento social e imagem corporal após a mastectomia:

Minha irmã hoje eu me sinto assim, normal, eu não me envergonho, as vezes eu saio sem esse bojo, tô cansada de sair, só que as vezes as pessoas olham bem pra mim, aí eu coloco assim (gesto que tenta esconder com

o braço), é vergonhoso, não por mim mas por causa das pessoa, mas eu mesmo não, eu não me envergonho de jeito nenhum, nada. (Girassol, 58 anos).

Pelo menos depois que eu fiz a cirurgia eu não tive nenhum preconceito de ninguém, muito pelo contrário, eu tive apoio, mas muito apoio mesmo, muito apoio.

[...] Às vezes me dá um pouco de incomodo quando eu vou sair, que tem que vestir a roupa, tem que vestir o sutiã com enchimento pra poder sair... se eu vou na esquina tem que vestir porque não posso sair assim, isso incomoda um pouco, mas eu não nego que quando olho no espelho eu penso: "poxa vida, eu tive que perder minha mama, ficar assim, mas depois quando reconstruir volta tudo de novo, eu tenho que agradecer a Deus porque tô viva! (Tulipa, 52 anos).

Eu não ligo muito, eu acho que questão estética, independentemente de ser mastectomia ou não, se você é gordo ou magro, porque eu sou gordinha, o problema nunca é a gente é o outro, as cobranças são dos outros

(Erva Doce, 28 anos).

[...]não saio de casa, é muito triste, hoje a gente passa nos cantos as pessoas ficam olhando pra gente. Eu não saio, só vivo dentro de casa, não saio nem no portão

(Orquídea, 56 anos)

O que se observa em relação às entrevistadas, são elas se sentiam incomodadas de alguma forma, pelo fato de terem perdido a mama. Em mulheres submetidas à mastectomia, na maioria das vezes sintomas de ansiedade e depressão tem um impacto negativo na avaliação da qualidade de vida das mesmas, principalmente no que se refere a ter que conviver com a ausência da mama ${ }^{25}$.
Em muitos casos, depois da cirurgia, a mulher se sente incompleta e marginalizada, pois a ausência do órgão é algo devastador. Muitas aceitam a perda do seio por ser o único modo para a cura, enquanto para outras, essa situação é traumática. Nesse momento, os acontecimentos ocorridos na sua vida, influenciam positiva ou negativamente na aceitação da nova imagem ${ }^{26}$.

\section{Conclusão}

Conclui-se que a realização da mastectomia, afeta consideravelmente a vida emocional das mulheres que precisam passar por esse procedimento, nesse sentido é de grande relevância que todas as pacientes diagnosticadas com câncer de mama tenham um adequado suporte psicológico após a realização do mesmo, principalmente por parte da família e dos profissionais de saúde que a acompanham.

Para algumas mulheres a vivência frente à mastectomia pode ser tranquila, no entanto para outras esse processo pode desencadear inúmeros sentimentos negativos sobre si mesma, que podem interferir de certa maneira na sua qualidade de vida. De alguma forma, passar por esse processo pode afetar os relacionamentos das mesmas, e é exatamente nesse ponto que o apoio familiar é importante para a aceitação da sua nova realidade e a capacidade de se superar frente os desafios ocasionados pela realização da remoção da mama.

Sendo assim, recomenda-se que as ações desenvolvidas pela equipe multiprofissional frente à mulher mastectomizada, tenha uma maior ênfase nos aspectos emocionais da mesma, uma vez que essa é umas das áreas mais afetadas frente a esse processo.

\section{Contribuição das autoras}

Pereira EP, Tavares EA, Queiroz GJC e Da Costa RSL participaram da concepção, delineamento, coleta de dados da pesquisa, interpretação dos dados, busca e análise estatística dos dados, redação do artigo científico. Quinteiro J e Ribeiro MAS participaram da concepção, delineamento, interpretação dos resultados e escrita científica. 


\section{Conflitos de Interesses}

Nenhum conflito financeiro, legal ou político envolvendo terceiros (governo, empresas e fundações privadas, etc.) foi declarado para nenhum aspecto do trabalho submetido (incluindo mas não limitando-se a subvenções e financiamentos, participação em conselho consultivo, desenho de estudo, preparação de manuscrito, análise estatística, etc.).

\section{Referencias}

1. Instituto Nacional do Câncer José Alencar Gomes da Silva. Conceito e Magnitude do câncer de mama.[Internet]. 2019. [acesso em 17 de set. 2019]. Disponível em: https://www.inca.gov. br/controle-do-cancer-de-mama/conceito-e-magnitude.

2. Instituto Nacional de Câncer José Alencar Gomes da Silva. Ministério da Saúde. A mulher e o câncer de mama no Brasil. 3.ed. Rio de Janeiro: INCA; 2014. p. 46.

3. Furlan VLA, Sabino Neto M, Abla LEF, Oliveira CJR, Lima AC, Ruiz BFO et al. Qualidade de vida e autoestima de pacientes mastectomizadas submetidas ou não a reconstrução de mama. Rev Bras Cir Plást. 2013;28(2):264-269. doi: 10.1590/S1983$\underline{51752013000200016}$

4. Ferreira DB, Farago PM, Reis PE, Funghetto SS. Nossa vida após o câncer de mama: percepções e repercussões sob o olhar do casal. Rev Bras Enferm. 2011;64(3):536-544. doi: 10.1590/5003471672011000300018

5. Moran M, Schnitt S, Giuliano A, Harris J, Khan S, Horton J et al. Society of Surgical Oncology-American Society for Radiation Oncology Consensus Guideline on Margins for Breast-Conserving Surgery With Whole-Breast Irradiation in Stages I and II Invasive Breast Cancer. Int J Radiat Oncol Biol Phys. 2014;88(3):553-564. doi: 10.1016/j.ijrobp.2013.11.012

6. Silva GM, Marinho VL. Repercussões psicossociais em mulheres que sofreram cirurgia de mastectomia acompanhadas pela liga feminina de combate ao câncer de mama de Gurupi-to. Rev Cereus. 2013;5(1):32-46.

7. Rocha CB, Fontenele GMC, Macêdo MS, Carvalho CMS, Fernandes MA, Veras JMMF et al. Sentimentos de mulheres submetidas à mastectomia total. Rev Cuid. 2018;10(1). doi: 10.15649/cuidarte.v10i1.606

8. Moura FMJSP, Silva MG, Oliveira SC, Moura LJSP. Os sentimentos das mulheres pós-mastectomizadas. Esc Anna Nery. 2010;14(3):477-484. doi: 10.1590/S1414-81452010000300007

9. Campos CJG. Método de análise de conteúdo: ferramenta para a análise de dados qualitativos no campo da saúde. Rev Bras Enferm. 2004;57(5):611-614. doi: 10.1590/50034$\underline{71672004000500019}$
10. Instituto Nacional do Câncer José Alencar Gomes da Silva. Ministério da Saúde. Estimativa 2016: Incidência de câncer no Brasil. [Internet]. 2018. [acesso em 20 de maio 2019]. Disponível em: https://www.inca.gov.br/campanhas/dia-nacional-decombate-ao-cancer/2015/estimativa-2016-incidencia-de-cancerno-brasil

11. Frutuoso RB, Macedo JQ, Araújo DV, Belém LF. Perfil de Mulheres Mastectomizadas no Município de Campina Grande-PB. In: XIII Encontro Latino Americano de Iniciação Científica, 2009; São Paulo: Universidade do Vale do Paraíba. 2009.

12. Gasparelo C, Sales CA, Marcon SS, Salci MA. Percepções de mulheres sobre a repercussão da mastectomia radical em sua vida pessoal e conjugal. Ciênc Cuid Saúde. 2010;9(3). doi: 10.4025/ cienccuidsaude.v9i3.12557

13. Ramos WSR, Sousa FS, Santos TR, Silva Júnior WR, França ISX, Figueiredo GCAL. Sentimentos vivenciados por mulheres acometidas por câncer de mama. J Health Sci Inst. 2012;30(3):241248.

14. Silva SED, Vasconcelos EV, Santana ME, Rodrigues ILA, Leite TV, Santos MLS et al. Representações sociais de mulheres mastectomizadas e suas implicações para o autocuidado. Rev Bras Enferm. 2010;63(5):727-734. doi: 10.1590/S003471672010000500006

15. Vale CCSO, Dias IC, Miranda KM. Câncer de mama: a repercussão da mastectomia no psiquismo da mulher. Mental. 2017; 11(21):527-45.

16. Fireman KM, Macedo FO, Torres DM, Ferreira FO, Lou MBA. Percepção das Mulheres sobre sua Funcionalidade e Qualidade de Vida após Mastectomia. Rev Brasileira de Cancerologia. 2019;64(4):499-508. doi: 10.32635/2176-9745.RBC.2018v64n4.198

17. Ferreira MLSM, Mamede MV. Representação do corpo na relação consigo mesma após a mastectomia. Rev LatinoAm Enfermagem. 2013;11(3):299-302. doi: 10.1590/S010411692003000300006

18. Silva GF, Bastos KD, Araujo AJS, Bispo TCF, Oliveira GRSA, Schulz RS. Mulheres submetidas à mastectomia: aspectos sentimentais e emocionais. Rev Enferm Contemp. 2018;7(1):72-80. doi: $10.17267 / 2317-3378$ rec.v7i1.1213

19. Viana JM, Campos LAL. Câncer de mama e mastectomia: cenário de atuação de enfermeiros. [Internet]. 2009. Disponível em: https://www.webartigos.com/artigos/cancer-de-mama-emastectomia-cenario-de-atuacao-de-enfermeiros/18331

20. Almeida TG, Comassetto I, Alves KMC, Santos AAP, Silva JMO, Trezza MCSF. Vivência da mulher jovem com câncer de mama e mastectomizada. Esc Anna Nery. 2015;19(3):432-438. doi: $\underline{10.5935 / 1414-8145.20150057}$ 
21. Fernandes AFC, Bonfim IM, Araújo IMA, Silva RM, Barbosa

ICFJ, Santos MCL. Significado do cuidado familiar à mulher mastectomizada. Esc Anna Nery. 2012;16(1):27-33. doi: 10.1590/ $\underline{\text { S1414-81452012000100004 }}$

22. Rodrigues NS, Orsini MRCA, Tertuliano IW, Bartholomeu D, Machado AA, Montiel,JM. O impacto da mastectomia na sexualidade da mulher. Lecturas: Educación Física y Deportes. 2018;23(242).

23. Pereira TIMM, Silva CRDV, Galiza DDF, Silva BN, Alencar RM, Véras GCB. Mastectomia e o sistema de enfrentamento feminino: nuances do apoio social e familiar. Revista Enfermagem Atual. 2019;87(Especial).

24. Faria NC, Fangel LMV, Almeida AM, Prado MAS, Carlo MMRP. Ajustamento psicossocial após mastectomia-um olhar sobre a qualidade de vida. Psic, Saúde \& Doenças. 2016;17(2);201-213. doi: $\underline{10.15309 / 16 p s d 170208}$

25. Kamińska M, Ciszewski T, Kukiełka-Budny B, Kubiatowski T, Baczewska B, Makara-Studzińska $\mathrm{M}$ et al. Life quality of women with breast cancer after mastectomy or breast conserving therapy treated with adjuvant chemotherapy. Ann Agric Environ Med. 2015;22(4);724-730. doi: 10.5604/12321966.1185784

26. Machado DL. Sendo companheiro de uma mulher mastectomizada: buscando ferramentas para a adaptação [dissertação]. Rio de Janeiro: Universidade do Estado do Rio de Janeiro; 2006. 